Journal of

Synchrotron

Radiation

ISSN 0909-0495

Received 1 March 2005

Accepted 4 March 2005

\title{
Towards an understanding of radiation damage in cryocooled macromolecular crystals
}

\author{
Colin Nave ${ }^{a *}$ and Elspeth F. Garman ${ }^{b}$ \\ ${ }^{a}$ CCLRC Daresbury Laboratory, Warrington WA4 4AD, UK, and ${ }^{\mathbf{b}}$ Laboratory of Molecular \\ Biophysics, Department of Biochemistry, Oxford University, Rex Richards Building, South Parks \\ Road, Oxford OX13QU, UK. E-mail: c.nave@dl.ac.uk
}

\begin{abstract}
Interest in radiation damage is growing rapidly owing to the surge in macromolecular crystallography experiments carried out at modern brilliant synchrotron macromolecular crystallography beamlines. Work on the characterization of radiation damage in cryocooled protein crystals is starting to have some impact on our understanding of the problem and of how damage might be affecting both the process of structure solution and the actual structure obtained. A brief review of the most recent developments is given together with an assessment of the remaining problems. Although progress is being made, the understanding of radiation damage is far from complete. Methods for recognizing the damage and treating the data are being made available but they are still at an early stage of development.
\end{abstract}

(C) 2005 International Union of Crystallography Printed in Great Britain - all rights reserved

Radiation damage is an important issue for macromolecular crystallographers, especially those trying to collect data from small crystals using intense $\mathrm{X}$-ray sources. This damage occurs at cryotemperatures (around $100 \mathrm{~K}$ ) and leads to a loss of resolution in the diffraction pattern. Even before significant resolution loss occurs, identifiable structural damage can be observed at specific sites in the protein. Early investigations carried out at room temperature (Blake \& Phillips, 1962) indicated that the damage was proportional to the absorbed dose (measured in Gray, $\mathrm{Gy}=\mathrm{J} \mathrm{kg}^{-1}$ ). After correction for the overall decay of crystalline order, they found that some reflections increased in intensity with X-ray exposure and deduced that this was probably due to specific sites of damage. However, the main observable effect at room temperature is to conceal the specific damage under a loss in resolution, i.e. diffractive power. Specific structural damage at cryogenic temperatures was largely unrecognized until systematic studies at third-generation synchrotron sources revealed that specific sites are vulnerable (Weik et al., 2000; Burmeister, 2000; Ravelli \& McSweeney, 2000). Taking the dose estimate of Blake \& Phillips (1962) and comparing it with measurements performed on ID14-4 at the ESRF (Owen et al., 2005), it can be computed that up to 70 times more absorbed dose can be delivered to a cryocooled macromolecular crystal compared with one at room temperature before significant degradation in crystal order occurs. Thus cryocooled protein crystals can preserve crystalline diffraction at a much higher dose than they would have done in the past when roomtemperature data collection was the norm. Under these high- dose conditions, the vulnerable sites must be inspected carefully when assigning structural or functional significance; in particular, active sites can be highly susceptible to damage (Weik et al., 2000).

It is important, for two reasons, to make an estimate of the dose which the crystal will receive during an experiment. Firstly, it allows the data collection to be planned in a way that minimizes the dose and maximizes the information obtained. This could, for example, be important in the case of phasing using anomalous scattering techniques. Secondly, it can be used to flag the possibility that specific changes may have occurred in the crystal at a level at which they could become observable. The dose absorbed by the crystal can be computed to a reasonable degree of accuracy from a knowledge of the incident-beam conditions (flux density, energy, shape and size) and the crystal constituents (Murray et al., 2004). However, estimating the incident X-ray intensity is problematic. Calibrated photodiodes or ion chambers can be used to find the flux through an aperture and this will allow a good estimate of the dose provided the beam intensity distribution at the sample position is known. The aperture size is not always well calibrated and, in any case, the apertures are some distance upstream of the sample. Significant structure can be present in the profile of the focus and the ideal flat or Gaussian profile does not always occur (Arzt et al., 2005). This can lead to some parts of the crystal receiving a much higher dose than other parts, even if an attempt has been made to match the illumination to the size of the crystal. A systematic study of the effects of the beam size illuminating the crystal has been 
carried out by Schulze-Briese et al. (2005). The authors report that a tightly focused beam results in a better signal-to-noise ratio but can lead to increased damage (or fewer data per dose unit) if only part of the crystal is irradiated. They showed that the rotation of a thick crystal about a horizontal axis during data collection can spread out the radiation load on the crystal from a small vertically focused beam, although a part on the rotation axis will continue to receive a high dose.

This result is an example of the compromises which often have to be made during data collection. Ideally, the beam should be matched to the crystal size (at the crystal) and be smaller than or matched to the detector point spread function (at the detector). Spreading the beam over the crystal minimizes radiation damage, while reducing the size of the diffraction spots at the detector maximizes the signal-to-noise ratio. The benefits of this arrangement for virus crystallography (large crystals and large unit cells) at room temperature have been well illustrated (Wikoff et al., 2000). A flexible variable focus arrangement of the type required is difficult to arrange in practice. However, a combination of an approximately parallel beam from an undulator and good slits to match the beam size to the crystal, can also be used. The majority of protein crystals examined on undulator sources have dimensions smaller than, or comparable with, the detector point spread function, so this arrangement gives a good compromise. It is an example of optimizing the beam on the sample rather than maximizing the beam intensity.

Even if a good estimate of the dose can be obtained, the effects on observable radiation damage cannot be easily predicted. Susceptibility of disulphide and carboxyl groups can depend on their environment in the protein or crystal (Ravelli \& McSweeney, 2000). The actual lifetime of a crystal could become shorter in those cases where specific damage breaks down crucial crystal contacts (Murray et al., 2005).

The effect of radiation damage is particularly problematic for phasing using anomalous scattering. Post mortem analysis of MAD data sets often reveals that better maps can be obtained using the single-wavelength data measured at the peak of the X-ray fluorescence. As a result (Jiang \& Sweet, 2004), single-wavelength techniques (SAD) are becoming more popular. There is no alternative to this approach when the absorption edge itself is inaccessible on the instrument (as is normally the case for anomalous scattering from sulfur and phosphorus).

Solvent flattening is a powerful method of breaking the phase ambiguity and is usually a critical factor in the success of structure solution by SAD. The solvent content of the crystal should therefore be a factor in the decision of whether or not to adopt single-wavelength or multiwavelength phasing methods. Two papers (Ravelli et al., 2005; González et al., 2005) are concerned with crystals which have a low solvent content, but the researchers adopt different approaches. Ravelli et al. (2005) have carefully evaluated the balance between high redundancy and radiation damage. The combination of optimized interpolation methods to extract the best anomalous signal together with modelling of the radiation damage is discussed for use in the RIPAS (radiation damage induced phasing with anomalous signal) method of structure solution at a single wavelength (Ravelli et al., 2003; Evans et al., 2003; Zwart et al., 2004). The paper by González et al. (2005) presents a slightly different point of view, and covers the use of two wavelengths, at the inflexion point and a position remote from the absorption edge. Having data which have been collected at the two different wavelengths breaks the phase ambiguity present with single-wavelength measurements. By avoiding data collection at the peak wavelength, absorption by the specimen is lower and consequent radiation damage is reduced.

Our understanding of the physical and chemical processes which occur during illumination of a cryocooled protein crystal with X-rays is gradually improving but is still rather rudimentary. Radiation chemistry studies are usually performed at a dose which is around 500 to 1000 times lower than that suffered by a macromolecular crystal in an X-ray crystallography experiment. The study by Terryn et al. (2005) examines the free radicals formed at various temperatures when solid insulin and lysozyme are irradiated with both electrons and $\gamma$-rays. The free radicals were characterized by EPR and the final degradation products by chromatography and electrophoresis. The EPR results indicated that the RSS ${ }^{\bullet}$ perthiyl radical is formed, but no compounds produced by bond breakage in its formation were identified in the product analysis. From these results and the fact that the EPR signals decay with time, the authors conclude that rearrangements have taken place between formation of the radicals and analysis of the products.

Macromolecular crystallographers are using increasingly smaller crystals for their structural studies. This is partly driven by the adoption of robotic methods which can dispense drops smaller than $100 \mathrm{~nL}$ for crystallization trials. Several authors (González \& Nave, 1994; Glaeser et al., 2000; Teng \& Moffat, 2000; Sliz et al., 2003) have estimated the smallest size of protein crystal from which it should be possible to collect $\mathrm{X}$-ray data (either a complete data set or a single diffraction image). This is dependent on both resolution and unit-cell size. Glaeser et al. (2000) suggest a conservative rule of thumb: that one high-resolution (in their case $2 \AA$ ) diffraction pattern, of $1^{\circ}$ rotation, can be obtained from a protein crystal if the crystal size (in micrometers) is one-tenth of the unit-cell size (in $\AA$ ). This gives a minimum dimension of $5 \mu \mathrm{m}$ for a $50 \AA$ unit cell. The path lengths of photoelectrons (energy 10-30 keV) in protein crystals are comparable with this dimension. If the photoelectrons leave the crystal, the deposition of energy in the crystal will be reduced. Nave \& Hill (2005) conclude that this could give a significant advantage for crystals of less than $5 \mu \mathrm{m}$ in size, especially if higher-energy (e.g. $30 \mathrm{keV}) \mathrm{X}$-rays were used for data collection. However, this advantage has still to be demonstrated in a practical situation, a necessary pre-requisite if this consideration is to drive the development of higher-energy beamlines for protein crystallography. In the absence of this effect, it appears that, away from any absorption edges, radiation damage is only weakly dependent on the X-ray wavelength used. The study at two wavelengths $(1.0 \AA$ and $2.0 \AA$ ) by Weiss et al. (2005) illustrates this: it 
included monitoring of specific damage at disulphide, methionine and cadmium sites.

An understanding of the processes which occur during cryocooling may lead to better cryoprotocols, an issue intimately related to radiation damage. The main detrimental effect on cooling protein crystals to cryotemperatures is that it often induces a decrease in crystalline order. This can manifest itself in an increase in the apparent mosaicity determined during a diffraction experiment. The paper by Weik et al. (2005) reports experiments on trigonal and orthorhombic trypsin crystals which were warmed up in a controlled stepwise manner and characterized at a series of temperatures from $100 \mathrm{~K}$ to $200 \mathrm{~K}$. As expected from previous work, the unit cell expanded as a function of temperature, but a sharp decrease of cell volume was observed at $195 \mathrm{~K}$ and $185 \mathrm{~K}$ in the two space groups, respectively, which was interpreted to be due to ultraviscous liquid solvent leaving the crystal and resulting in ice rings on the diffraction patterns. The presence of liquidlike solvent links with other recent studies of annealing mechanisms (Kriminski et al., 2002; Juers \& Matthews, 2004) and is pertinent to studies of both specific radiation damage as a function of temperature and for the freeze-trapping of intermediate states in the investigation of enzymatic mechanisms (Ursby et al., 2002).

A recurrent debate in radiation damage discussions revolves around whether or not there are dose-rate effects at cryotemperatures. It can be imagined that these could occur if the more intense X-ray beams at third-generation synchrotron sources cause a steep increase in temperature of the protein crystal. Sophisticated modelling of the heat-transfer processes is being carried out to investigate whether this effect might occur. The paper by Mhaisekar et al. (2005) reports the use of computational fluid dynamics to calculate the non-uniform convective heat-transfer coefficients around a cryocooled crystal. The effects of varying the gas velocity and properties, the crystal size and thermal conductivity, and the incidentbeam intensity and size have all been modelled. The authors conclude that there is little temperature variation within an irradiated crystal and that the largest change (between 5 and $10 \mathrm{~K}$ for the systems investigated) is at the interface of the crystal/loop system and the gas stream. These results imply that there should be no large differential thermal diffusion gradients within a crystal, and that these intense beams should not take the crystal above the glass transition temperature around $155 \mathrm{~K}$ (Weik et al., 2001).

Many fundamental studies regarding radiation damage are still required. For instance, although the issue of heating now appears to be well modelled, experimental verification is not yet available. Also, investigations on the susceptibility of particular atom types, for example using radiation above and below their absorption edges, would be particularly interesting. This should shed light on the relative contributions of initial absorption and subsequent processes. The reason for the different susceptibility of disulphide linkages and carboxyl groups is not yet entirely clear. Each photoelectron takes part in several hundred inelastic scattering processes in which bonds could be broken and radicals created. The susceptibility of particular bonds to breakage via such interactions is worth investigating. Once the bond is broken, small preferential movements of the atoms have to occur for the changes (including disorder) to appear on electron density maps. More data are required both on the susceptibility of particular residues to specific damage and the dependency of this on their environment (e.g. buried, on surface, hydrated, relationship to folding). However, there is now enough data to give a useful indication of the dose at which resolution loss is likely to occur. It is more difficult to predict specific damage and the way such damage could affect experimental phasing although there are enough examples of specific damage to indicate which residues in a refined structure might be altered owing to radiation damage. A procedure for flagging such residues during structure reporting and deposition would be useful.

Strategy determination software incorporating estimates of radiation damage can only provide a guideline, as the effects are dependent on the detailed structure of the protein and the crystal. Monitoring the effects of radiation damage during data collection, so that the strategy can be modified, will also be required and software for carrying this out is also being developed (Popov \& Bourenkov, 2003). The data for developing this software have come from the experience (good and bad) gained over the past few years at high-intensity X-ray sources. Failed MAD and SAD experiments, for example, have provided very useful information for developing better data-collection strategies. However, it will be some time before the software for dealing with this matches the experience of expert crystallographers. An awareness of radiation damage is therefore very important for those collecting X-ray diffraction data as it can lead to more successful data collection and better interpretation of the results. There is no longer any good reason why a large number of MAD and SAD experiments should fail due to insufficient attention to radiation damage issues. In a review over two years ago (Garman \& Nave, 2002), it was stated that 'the X-ray structural biology community are aware of the issue and, like the electron microscopists, are designing their experiments to take account of the effect of intense doses on the specimens'. Since then, a further understanding of radiation damage has been acquired, and ways of treating the data and recognizing the specific effects of radiation damage are becoming available. However, this understanding is far from complete and the methods for recognizing the damage and treating the data are still at an early stage of development. It is therefore to be hoped that there will continue to be further investigations of the type reviewed in this paper.

We thank Raimond Ravelli for providing useful comments on this manuscript. We also gratefully acknowledge the generosity of the ESRF, the EU Access to Research Infrastructure Action of the Improving Human Potential Programme (MAX-INF project, HPRI-CT-2000-40021) and the EU IHP Access to Research Infrastructures Grant to EMBL-Grenoble (HPRI-CT-1999-00022) for providing funding to allow the Third International Workshop on X-ray 
Damage to Crystalline Biological Samples to be held at the ESRF, Grenoble in November 2003. The papers in this issue were presented at the workshop. The Organisers (E. Garman, S. McSweeney, C. Nave, R. Ravelli and G. Rosenbaum) hope to hold the fourth such workshop during 2006.

\section{References}

Arzt, S., Beteva, A., Cipriani, F., Delageniere, S., Felisaz, F., Forstner, G., Gordon, E., Launer, L., Lavault, B., Leonard, G., Mairs, T., McCarthy, A., McCarthy, J., McSweeney, S., Meyer, J., Mitchel, E., Monaco, S., Nurizzo, D., Ravelli, R., Rey, V., Shepard, W., Spruce, D., Svensson, O. \& Theveneau, P. (2005). Prog. Biophys. Mol. Biol. In the press.

Blake, C. C. F. \& Phillips, D. C. (1962). Biological Effects of Ionizing Radiation at the Molecular Level, pp. 183-191, International Atomic Energy Agency Symposium, Brno, Czechoslovakia, 2-6 July 1962. Vienna: IAEA.

Burmeister, W. P. (2000). Acta Cryst. D56, 328-341.

Evans, G., Polentarutti, M., Djinovic Carugo, K. \& Bricogne, G. (2003). Acta Cryst. D59, 1429-1434.

Garman, E. \& Nave, C. (2002). J. Synchrotron Rad. 9, 327-328.

Glaeser, R., Facciotti, M., Walian, P., Rouhani, S., Holton, J., MacDowell, A., Celestre, R., Cambie, D. \& Padmore, H. (2000). Biophys. J. 78, 3178-3185.

Gonzalez, A. \& Nave, C. (1994). Acta Cryst. D50, 874-877.

González, A., von Delft, F., Liddington, R. C. \& Bakolitsa, C. (2005). J. Synchrotron Rad. 12, 285-291.

Jiang, J. \& Sweet, R. M. (2004). J. Synchrotron Rad. 11, 319-327.

Juers, D. H. \& Matthews, B. W. (2004). Acta Cryst. D60, 412-421.

Kriminski, S., Caylor, C. L., Nonato, M. C., Finkelstein, K. D. \& Thorne, R. E. (2002). Acta Cryst. D58, 459-471.
Mhaisekar, A., Kazmierczak, M. J. \& Banerjee, R. (2005). J. Synchrotron Rad. 12, 318-328.

Murray, J. W., Garman, E. F. \& Ravelli, R. B. G. (2004). J. Appl. Cryst. 37, 513-522.

Murray, J. W., Rudiño-Piñera, E., Owen, R. L., Grininger, M., Ravelli, R. B. G. \& Garman, E. F. (2005). J. Synchrotron Rad. 12, 268-275. Nave, C. \& Hill, M. A. (2005). J. Synchrotron Rad. 12, 299-303.

Owen, R. L., Rudiño-Piñera, E. \& Garman, E. (2005). In preparation.

Popov, A. N. \& Bourenkov, G. P. (2003). Acta Cryst. D59, 1145-1153.

Ravelli, R. G. B., Leiros, H. K. S., Pan, B., Caffrey, M. \& McSweeney, S. (2003). Structure, 11, 217-224.

Ravelli, R. G. B. \& McSweeney, S. (2000). Structure, 8, 315-328.

Ravelli, R. B. G., Nanao, M. H., Lovering, A., White, S. \& McSweeney, S. (2005). J. Synchrotron Rad. 12, 276-284.

Schulze-Briese, C., Wagner, A., Tomizaki, T. \& Oetiker, M. (2005). J. Synchrotron Rad. 12, 261-267.

Sliz, P., Harrison, S. C. \& Rosenbaum, G. (2003). Structure, 11, 13-19.

Teng, T.-Y. \& Moffat, K. (2000). J. Synchrotron Rad. 7, 313-317.

Terryn, H., Deridder, V., Sicard-Roselli, C., Tilquin, B. \& HouéeLevin, C. (2005). J. Synchrotron Rad. 12, 292-298.

Ursby, T., Weik, M., Fioravanti, E., Delarue, M., Goeldner, M. \& Bourgeois, D. (2002). Acta Cryst. D58, 607-614.

Weik, M., Kryger, G., Schreurs, A. M. M., Bouma, B., Silman, I., Sussman, J. L., Gros, P. \& Kroon, J. (2001). Acta Cryst. D57, 566573.

Weik, M., Ravelli, R. G. B., Kryger, G., McSweeney, S., Raves, M. L., Harel, M., Gros, P., Silman, I., Kroon, J. \& Sussman, J. L. (2000). Proc. Natl. Acad. Sci. 97, 623-628.

Weik, M., Schreurs, A. M. M., Leiros, H.-K. S., Zaccai, G., Ravelli, R. G. B. \& Gros, P. (2005). J. Synchrotron Rad. 12, 310-317.

Weiss, M. S., Panjikar, S., Mueller-Dieckmann, C. \& Tucker, P. A. (2005). J. Synchrotron Rad. 12, 304-309.

Wikoff, W. R., Schildkamp, W. \& Johnson, J. E. (2000). Acta Cryst. D56, 890-893.

Zwart, P. H., Banumathi, S., Dauter, M. \& Dauter, Z. (2004). Acta Cryst. D60, 1958-1963. 